\title{
Clotting time analysis of citrated blood samples is strongly affected by the tube used for blood sampling
}

\author{
Sofia Ramström
}

\section{Linköping University Post Print}

N.B.: When citing this work, cite the original article.

Original Publication:

Sofia Ramström, Clotting time analysis of citrated blood samples is strongly affected by the tube used for blood sampling, 2005, Blood Coagulation and Fibrinolysis, (16), 6, 447-452.

Copyright: Lippincott, Williams \& Wilkins http://www.lww.com/

Postprint available at: Linköping University Electronic Press http://urn.kb.se/resolve?urn=urn:nbn:se:liu:diva-31049 
MBC200022

Received 27 December 2004 Revised 21 May 2005 Accepted 6 June 2005

Blood Coagulation \& Fibrinolysis

Blood collection tubes and coagulation analysis Ramström

Technical note

Sponsorship: This study was supported by the Swedish Research Council grant K200471X-15060-01A and the County Council of Östergötland.

Clotting time analysis of citrated blood samples is strongly affected by the tube used for blood sampling

Sofia Ramström

Department of Biomedicine and Surgery, Division of Clinical Chemistry, University Hospital, Linköping, Sweden

Correspondence and requests for reprints to Sofia Ramström, Department of Biomedicine and Surgery, Division of Clinical Chemistry, University Hospital, SE-581 85 Linköping, Sweden 
Tel: +4613222425

Fax: +4613223240

E-mail: sofra@ibk.liu.se

Our aim was to establish whether differences in clotting times for recalcified blood and plasma samples might be explained by the use of different blood collection tubes. Samples obtained from different plastic vacuum tubes were recalcified and clotting times determined by free oscillation rheometry. The clotting times for blood collected in Vacutainer (Becton Dickinson, Rutherford, New Jersey, USA) or Vacuette (Greiner Bio-One, Kremsmünster, Austria) tubes decreased with time, with maximal effect after 30 min. Blood from Monovette (Sarstedt, Nümbrecht, Germany) tubes displayed longer clotting times, which did not decrease with time. Clotting times for plasma prepared after $1 \mathrm{~h}$ storage in Vacutainer or Vacuette tubes were unaffected by subsequent addition of corn trypsin inhibitor to inhibit factor XIIa, although an antibody against factor XI prolonged the clotting time markedly. In Monovette plasma, both corn trypsin inhibitor and anti-factor XI effectively prolonged the clotting time. When corn trypsin inhibitor or anti-factor XI was added to the tubes before blood collection, but both additions clearly prolonged the clotting times in all types of tubes, even though corn trypsin inhibitor was less effective in whole blood. Antibodies against human tissue factor did not affect the clotting times. The amounts of platelet or leukocyte microparticles in plasma were low and similar in all tubes. This indicates that blood collection in Vacutainer or Vacuette tubes induces a rapid activation of factor XII and factor XI. 


\section{Keywords}

blood coagulation tests; blood specimen collection; citrates; factor XIa; factor XIIa; whole blood coagulation time

\section{Introduction}

Coagulation analysis of blood and plasma is usually performed using citrate anticoagulated blood, which is recalcified at the time of analysis. In most common coagulation assays used today, like ACT, APTT and PT $\{\mathbf{1}\}$, the coagulation is also accelerated by the addition of some reagent, which shortens the clotting time for the sample to the range of seconds. Increasing knowledge of the process of coagulation in vivo, however, has shown that this is a process normally requiring minutes, and which occurs without a complete consumption of the plasma coagulation factors present $[1,2]$. This has led to the development and description of several methods by which whole blood clotting times or thrombin generation are measured during several minutes. Some of these methods, like thromboelastography, ROTEM and Sonoclot $\{2\}$, have been used in various settings during anaesthesiology and surgery, while others, like free oscillation rheometry (FOR) and thrombin generation measurements are, as yet, mostly used for laboratory investigations. In our studies of such systems and during the literature search for similar studies, we have noticed that while we, as well as others [3-5], usually obtained clotting times exceeding 20 min for citrated blood samples after recalcification, other reports were stating that this time was normally 6-10 min [6]. We further noticed that differing results have been reported regarding the use of citrated blood samples compared with native ones in thromboelastography, while some studies state that there are no differences [4,5], others report that citrated samples differ 
substantially from native blood samples $[3,7,8]$. The aim of this study was to establish whether some of these differences might depend on pre-analytic factors such as the use of different blood collection systems.

\section{Methods}

Tri-sodium citrate and $\mathrm{CaCl}_{2} \times 2 \mathrm{H}_{2} \mathrm{O}$ were from E. Merck, Darmstadt, Germany. Corn trypsin inhibitor (CTI) was from Enzyme Research Laboratories (South Bend, Indiana, USA). The monoclonal antibodies against human tissue factor (\#4509 and \#4503) were from American Diagnostica Inc. (Greenwich, Connecticut, USA). The polyclonal chicken antibody (IgG fraction purified from egg yolk) directed against human factor XI was a kind gift from Anders Larsson at the Department of Clinical Chemistry, Uppsala University Hospital, Sweden.

The blood donors were donating blood at Linköping University Hospital and fulfilled the criteria that the hospital sets for the blood donors. Whole blood samples were collected in different plastic vacuum tubes, Vacutainer (Becton Dickinson, Rutherford, New Jersey, USA), Vacuette (Greiner Bio-One, Kremsmünster, Austria) or SMonovette (Sarstedt, Nümbrecht, Germany), all containing sodium citrate (129 mmol/l, i.e. $2.8 \%, \mathrm{pH}$ around 5.5, final dilution 1:10). In one set of experiments, the tubes were pre-filled with corn trypsin inhibitor (CTI) (final concentration $30 \mu \mathrm{g} / \mathrm{ml}$ ), to inhibit factor XIIa (fXIIa), or antibodies towards human factor XI or tissue factor (final concentrations $33 \mu \mathrm{g} / \mathrm{ml}$ and $10 \mu \mathrm{g} / \mathrm{ml}$, respectively). 
The blood was stored at room temperature and was recalcified after different times of incubation. For some experiments the blood was centrifuged at $2500 \mathrm{~g}$ for $15 \mathrm{~min}$ to obtain platelet poor plasma. This plasma was either recalcified directly or incubated for 5 min at $37^{\circ} \mathrm{C}$ with $\mathrm{CTI}$ or antibodies towards human factor XI or tissue factor before recalcification (final concentrations $50,55 \mu \mathrm{g} / \mathrm{ml}$ and $17 \mu \mathrm{g} / \mathrm{ml}$, respectively; the higher concentrations were used to achieve approximately the same plasma concentrations as in the experiments when the inhibitors were added directly to the blood collection tubes). In cases when sub-sampling for plasma preparation or incubation had to be performed, standard plastic tubes of polypropylene or polystyrene, that had been previously tested not to affect the clotting times, were used. The time of contact with these other tubes was also minimized. Calcium chloride $(0.5 \mathrm{~mol} / \mathrm{l})$ was added to a final concentration of $10 \mathrm{mmol} / \mathrm{l}$ in blood and $18 \mathrm{mmol} / \mathrm{l}$ in plasma, which gave a calcium ion activity of $1.2 \mathrm{mmol} / \mathrm{l}$ in serum after clotting (determined potentiometrically with an ICA 2 Ionised Calcium Analyser from Radiometer, Copenhagen, Denmark).

Clotting of recalcified blood and plasma samples was measured utilizing free oscillation rheometry (FOR). The four-channel free oscillation rheometers, ReoRox4, disposable plastic sample cups and software (ReoRox4 v2.00 and ReoRox4Viewer v2.01) were from MediRox AB, Nyköping, Sweden. This technique has been used by us in previous studies, and its close correlation to the clotting time determined by visual inspection has been verified [9]. Plasma samples still not clotted after 3 hours were assigned a clotting time of $180 \mathrm{~min}$. In the inhibitor experiments, all samples from a donor were excluded if the control sample, to which no inhibitor had been added, did not clot within 180 minutes. 
The presence of platelet and leukocyte microparticles within plasma was investigated by flow cytometry, utilising a Coulter Epics XLFlow Cytometer (Beckman Coulter Inc., Fullerton, California, USA). Phycoerythrin-conjugated mouse antibodies against GPIIb (CD41) and phycoerythrin-conjugated $\operatorname{IgG}_{1}$ (negative control) were purchased from Dako AS (Glostrup, Denmark). A PC5-conjugated mouse antibody against leukocyte antigen CD45 was purchased from Immunotech (Marseille, France). In the protocol, particles were collected using forward and side scatter. The scattergram contained a gate in forward scatter for the determination of microparticles, which contained $1-2 \%$ of the particles in a normal platelet-rich plasma sample. The number of phycoerythrin or PC5positive particles collected within this gate in $180 \mathrm{~s}$ was determined for platelet poor plasma samples, collected by centrifugation (at $2500 \mathrm{~g}, 15 \mathrm{~min}$ ) from the different vacuum tubes $1 \mathrm{~h}$ after blood collection. Most samples were run in duplicate.

\section{Statistics}

Due to the rather small sample sizes and the large differences in variance between different time points, we chose to use the Friedman test, a non-parametric alternative to the repeated measures analysis of variance. The clotting times for blood or plasma samples with and without inhibitors added were compared with the Friedman test in combination with a post-hoc-test (based on a derivation of the distribution of the largest difference between the results with and without inhibitor and a comparison of rank sums between the different groups compared) for the cases involving repeated measurements, and with the non-parametric Wilcoxon signed ranks test in the other cases. Statistical analyses were performed using SPSS for Windows (SPSS Inc., Chicago, Illinois, USA) 
except for the Friedman post-hoc test, which was performed by a statistician at Linköping University.

\section{Results}

Blood collected in Vacutainer or Vacuette tubes displayed markedly shorter clotting times than blood collected in Monovette tubes (Fig. 1). This was not due to the anticoagulant solution, since the clotting time was still prolonged for samples in which citrate solution from a Vacutainer tube had been transferred to a Monovette tube. The clotting times for blood from Vacutainer or Vacuette tubes decreased with incubation time, with maximal effect after approximately $30 \mathrm{~min}$, indicating that eventual differences in vacuum during blood sampling was not the determinant factor.

Blood samples were also sub-sampled and centrifuged at different time points to obtain platelet poor plasma. When comparing clotting times for these plasma samples, it was shown that even though the times for plasma from Vacutainer, Vacuette and Monovette tubes were similar after 15 min, they rapidly decreased in the Vacutainer and Vacuette tubes, while the times for plasma from the Monovette tubes stayed almost unchanged (Fig. 2).

The clotting time decrease with time observed for some blood collection tubes made us suspect that the contact with the tubes might have caused activation of factor XII from the intrinsic coagulation pathway. The other possibility was coagulation activation via the extrinsic pathway, which would imply that tissue factor had, by some means, been exposed by the blood cells present. To examine these different possibilities, plasma was 
prepared from whole blood stored for $1 \mathrm{~h}$ in the different vacuum tubes. CTI or antibodies against factor XI or tissue factor was added to this plasma.

The results are shown in Fig. 3. Inhibition of fXIIa by CTI had minor effects on the clotting times for plasma samples from Vacutainer and Vacuette tubes. In the Monovette tubes, however, CTI was very effective in prolonging the clotting times. On the other hand, addition of an antibody against factor XI prolonged the clotting time significantly for plasma from all types of tubes.

The antibody against human tissue factor did not affect the clotting times in samples from Vacutainer tubes, the clotting time was $11.7 \pm 1.9$ min without and $11.9 \pm 1.1 \mathrm{~min}$ with the antibody (mean $\pm \mathrm{SD}, n=5$ ). For Vacuette tubes the clotting time was $17.8 \pm 11.1$ min without and 14.4 \pm 5.0 minutes with the antibody $(n=3)$. In samples from Monovette tubes the clotting times showed large variations, $44.9 \pm 38.7 \mathrm{~min}$ without and $69.8 \pm 61.7$ min with the antibody $(n=7)$, the difference was not significant. Another, non-inhibitory antibody against human tissue factor gave clotting times of $47.4 \pm 40.2 \mathrm{~min}$ and also prolonged clotting times in two out of four experiments, indicating that this might be an unspecific effect.

To check whether these effects were a consequence of the blood contacting the tubes, the inhibitors were also added to the tubes before blood collection (Fig. 4). The blood was left within the tubes for $1 \mathrm{~h}$ at room temperature before recalcification. In this setting, the presence of CTI indeed caused a prolongation of the clotting time of approximately $10 \mathrm{~min}$ in blood from all tubes. The presence of an antibody against 
factor XI, however, could completely prevent coagulation in four out of five samples from Vacutainer, three out of five samples from Vacuette and one out of five samples from Monovette tubes. Platelet poor plasma was also prepared from the same blood samples and recalcified (Fig. 5). It is obvious that in the absence of blood cells, both CTI and anti-factor XI are were very effective in prolonging the clotting time in all types of tubes, with a complete anticoagulation (no clotting within $180 \mathrm{~min}$ ) in 10 out of 15 experiments with CTI and 14 out of 15 experiments with anti-factor XI. No effects on the clotting time were seen when the inhibitory antibody against tissue factor was present in the tubes (data not shown).

The presence of activated platelets has previously been shown to shorten the clotting times [10]. The same shortening might possibly occur if the plasma contains microparticles released from platelets, since these have been shown to support coagulation via tissue factor-independent pathways [11]. Therefore we checked plasma samples from Vacutainer, Vacuette and Monovette tubes $(n=3)$ for the presence of platelet or leukocyte derived microparticles (particles positive for CD41 or CD45, respectively) by flow cytometry, but found no significant amounts of microparticles in plasma from any of the three brands of tubes, and the small numbers still detected did not differ between the different tubes (data not shown).

\section{Discussion}

The findings reported here indicate that blood and plasma collected in Vacutainer or Vacuette tubes become procoagulant with time in a process starting immediately after blood collection and with maximal effects after approximately $30 \mathrm{~min}$ of storage. 
When comparing the results from previous studies using thromboelastography, we found that those that did not show any large differences between native and citrated blood $[4,5]$ did not use vacuum tubes for the blood collection, while the studies reporting rapid changes towards a procoagulant state in citrated samples did use Vacutainer tubes $[7,8]$. This cannot be the only explanation, however, since another study [3] found significant differences between native and citrated blood, as well as an overall trend towards hypercoagulability in citrated blood samples during storage, but did not use vacuum tubes for the blood collection. In that study, the authors hypothesized that their findings were partly due to the fact that they were using thromboelastography without any coagulation activator, which increased its sensitivity towards these trends. It is reasonable to assume that all factors decreasing the clotting time also decrease the possibility to detect subtle changes in coagulability of the original sample. This should mean that the use of an unsuitable type of blood collection tube might be able to mask changes caused by, for example, inhibitors or weak agonists, just like the use of too strong coagulation activators. In these cases, the experiments performed might lead to the wrong conclusions.

The fact that CTI added to Vacutainer or Vacuette tubes before sample collection could inhibit the procoagulant effect indicates that this effect is due to activation of the intrinsic pathway, most probably due to blood contact with foreign surfaces. All manufacturers claim to use polypropylene plastic for the surface of the tube contacting the platelets. During manufacture of polypropylene products, however, different additives are often included, like flame retardants, antioxidizers, light stabilizers or 
clarifying agents. The use of different additives in the different tubes could possibly be one reason for the differences experienced. Another difference might be the material in the stoppers, which was not stated by any manufacturer. The results obtained when CTI was added to plasma samples after $1 \mathrm{~h}$ might, however, lead to false conclusions, since an addition at this stage had minor effects. In that stage, however, an antibody against factor XI still had a marked effect, showing that the fXIIa formed during storage activates factor XI even in the presence of citrate, in a mechanism similar to the one occurring during the preincubation phase of an APTT test, but without the addition of a negatively charged coagulation activator [12]. The antibody against tissue factor did not seem to affect the coagulation at any stages, indicating that the extrinsic pathway was not involved in the shortening of the clotting times.

It has been reported that magnesium contamination of the citrate solution in Vacutainer tubes could affect measurements of prothrombin time [13]. In our experiments, however, the differences could not be due to any additions to the Vacutainer citrate solution, since no clotting time decrease occurred when this solution was transferred to a Monovette tube. In the study mentioned above, the authors also used Vacutainer tubes made of siliconized glass instead of the plastic tubes used by us.

Another hypothesis was that the clotting time decrease was due to the formation of procoagulant microparticles when blood cells got into contact with the tubes. This hypothesis was based on two reports studying platelet-derived microparticles in citrate plasma from healthy volunteers, collected in Vacutainer tubes. Similar to our experiments, these particles were found to generate thrombin in a process involving 
factor XI, although largely independent of factor XII and tissue factor $[11,14]$. In an earlier study by the same group, much lower amounts of microparticles were found in plasma collected in ethylenediaminetetraacetic acid [15], which is just in line with a report by Wolf from 1967 [16]. We therefore analysed the presence of microparticles of platelet (CD41-positive) or leukocyte (CD45-positive) origin using flow cytometry, but found similar and very small amounts of microparticles in plasma from all three brands of tubes. It is therefore not probable that formation of microparticles explains the decrease in clotting times observed.

From this we conclude that blood collection in Vacutainer or Vacuette tubes will cause an activation of factor XII and XI during the first 30 min after blood collection, a fact that might have a severe impact on the results for assays in which clotting times (and probably other coagulation related endpoints, such as thrombin generation) in the range of several minutes are to be studied. For clinical routine analyses like APTT and PT, with clotting times in the range of seconds, the differences are certainly smaller, but they might still be relevant in samples for which clotting times are prolonged. Further studies are needed to answer that question. From our point of view, however, the Monovette system seems to be preferable, since the clotting times recorded with these tubes are similar to the ones we usually obtain from blood without any anticoagulant added [10].

\section{Acknowledgements}


I am very grateful to Professor Tomas L. Lindahl and the others involved in the Haemostasis group at Linköping University Hospital for discussions and for sharing of their expertise in the field. 


\section{References}

Mann K. Biochemistry and physiology of blood coagulation. Thromb Haemost 1999; 82:165-174.

2

Rand M, Lock J, van't Veer C, Gaffney D, Mann K. Blood clotting in minimally altered whole blood. Blood 1996; 88:3432-3445. 3 Zambruni A, Thalheimer U, Leandro G, Perry D, Burroughs AK. Thromboelastography with citrated blood: comparability with native blood, stability of citrate storage and effect of repeated sampling. Blood Coagul Fibrinolysis 2004; 15:103-107.

Assessment of blood coagulation in severe liver disease using thromboelastography: use of citrate storage versus native blood. Blood Coagul Fibrinolysis 2003; 14: 211-216. 5 Bowbrick VA, Mikhailidis DP, and Stansby G. The use of citrated whole blood in thromboelastography. Anesth Analg 2000; 90:1086-1088.

6

Ungerstedt J, Kallner A, Blombäck M. Measurement of blood and plasma coagulation time using free oscillating rheometry. Scand J Clin Lab Invest 2002; 62:135-140.

Vig S, Chitolie A, Bevan DH, Halliday A, Dormandy J.

Thromboelastography: a reliable test? Blood Coagul Fibrinolysis 2001; 12:555-561. 8 Camenzind V, Bombeli T, Seifert B, Jamnicki M, Popovic D, Pasch T, Spahn DR. Citrate storage affects Thrombelastograph analysis [see comments]. Anesthesiology 2000; 92:1242-1249. 
oscillation rheometry and visual inspection and a viscoelastic description of the clotting phenomenon. Scand J Clin Lab Invest 2003; 63:397-406. coagulation: effects of platelet agonists and of GPIIb/IIIa inhibitors studied by free oscillation rheometry. Thromb Res 2002; 105:165-172.

Cell-derived microparticles circulate in healthy humans and support low grade thrombin generation. Thromb Haemost 2001; 85:639-646.

12

Kogan AE, Kardakov DV, Khanin MA. Analysis of the activated partial thromboplastin time test using mathematical modeling. Thromb Res 2001; 101:299_ 310.

13 van den Besselaar AM, Rutten WP, Witteveen E. Effect of magnesium contamination in evacuated blood collection tubes on the prothrombin time test and ISI calibration using recombinant human thromboplastin and different types of coagulometer. Thromb Res 2005; 115:239-244. Sturk A. Microparticles from patients with multiple organ dysfunction syndrome and sepsis support coagulation through multiple mechanisms. Thromb Haemost 2001; $85: 810-820$.

15 Nieuwland R, Berckmans RJ, McGregor S, Boing AN, Romijn FP, Westendorp RG, et al. Cellular origin and procoagulant properties of microparticles in meningococcal sepsis. Blood 2000; 95:930-935. 
Br J Haematol 1967; 13:269-288.

Fig. 1 Clotting times for citrated blood from different blood collection tubes that was recalcified at different time points after blood collection. Each point shows the mean and SD for clotting times from five different individuals.

Fig. 2 Clotting times upon recalcification of platelet poor plasma anticoagulated with citrate, which was portioned and centrifuged at different time points after blood collection (15 min, $30 \mathrm{~min}$ or $1 \mathrm{~h}$ ). Each point shows the mean and SD for clotting times from five or six different individuals.

Fig. 3 Clotting times upon recalcification of platelet poor plasma that was prepared $1 \mathrm{~h}$ after blood collection. The different inhibitors (corn trypsin inhibitor (CTI; $50 \mu \mathrm{g} / \mathrm{ml}$ ) or an antibody against human factor XI (anti-fXI; $55 \mu \mathrm{g} / \mathrm{ml})$ ) were incubated with the plasma for 5 minutes at $37^{\circ} \mathrm{C}$ before recalcification. The bars show the mean and the error bars the SD. Asterisks shows significant changes when compared to the clotting times for the corresponding control samples without inhibitor $(* P<0.05$; ** $P<0.01)$. $\mathrm{TF},\{\mathbf{4}\}$

Fig. 4 Clotting times for whole blood where corn trypsin inhibitor (CTI; $30 \mu \mathrm{g} / \mathrm{ml})$ or an antibody towards human factor XI (anti-fXI; $33 \mu \mathrm{g} / \mathrm{ml}$ ) had been added to the vacuum tubes before blood sampling. Recalcification and clotting time determinations were 
done $1 \mathrm{~h}$ after blood collection. The bars show the mean and the error bars the SD. Asterisks show significant changes when compared with the clotting times for the corresponding control samples without inhibitor $(* P<0.05$; ** $P<0.01)$.

Fig. 5 Clotting times for platelet poor plasma when corn trypsin inhibitor (CTI;

$30 \mu \mathrm{g} / \mathrm{ml}$ ) or an antibody towards human factor XI (anti-fXI; $33 \mu \mathrm{g} / \mathrm{ml}$ ) had been added to the vacuum tubes before blood sampling. Plasma preparation, recalcification and clotting time determinations were performed $1 \mathrm{~h}$ after blood collection. The bars show the mean and the error bars the SD. Asterisks shows significant changes when compared to the clotting times for the corresponding control samples without inhibitor $(* P<0.05 ; * * P<0.01)$. 
Figure 1:

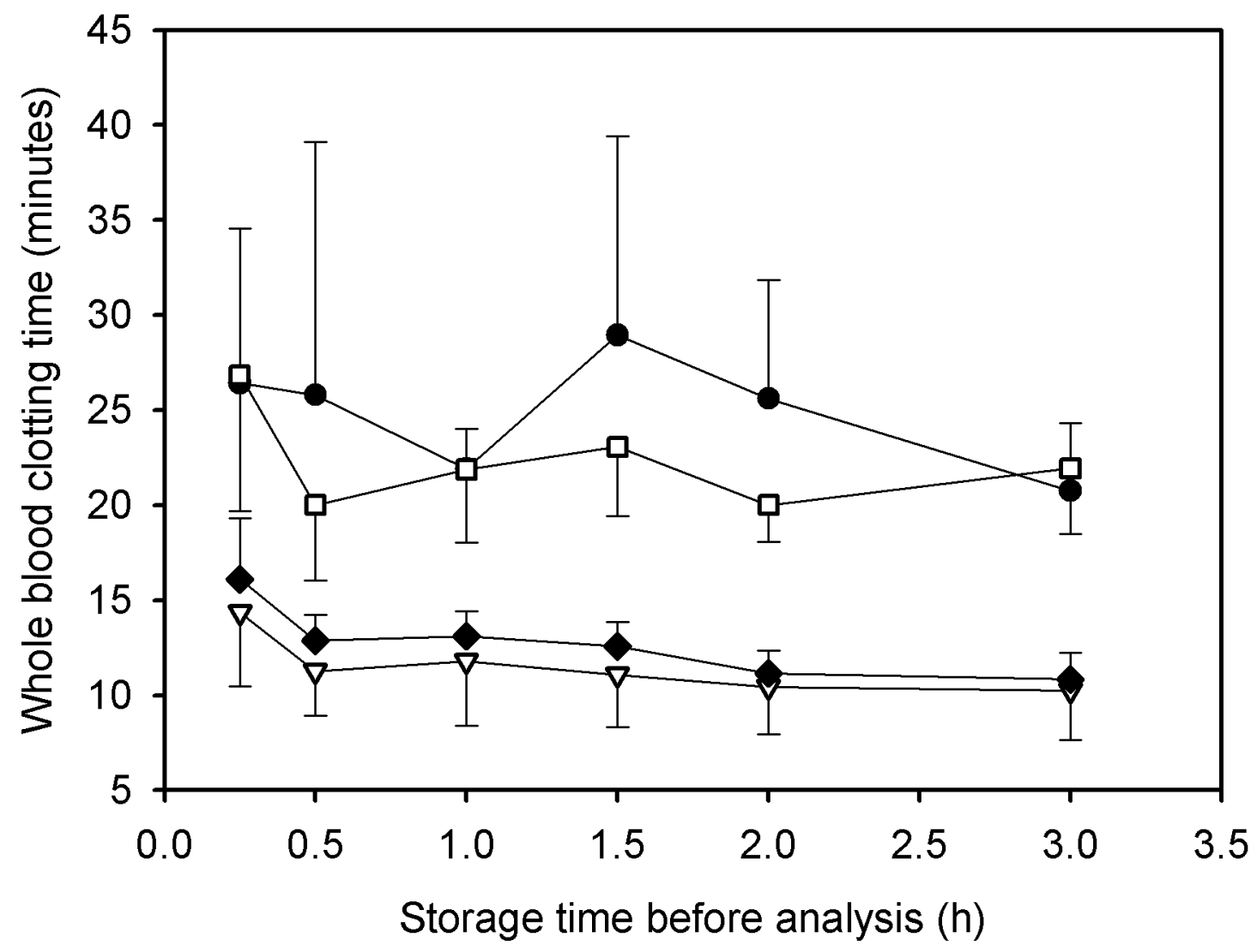

- Monovette tubes with Vacutainer citrate solution, pH $5.6(n=5)$

- 느 Monovette tubes with locally prepared citrate solution, pH $5.6(n=5)$

$P$ Vacutainer tubes $(n=5)$

$\checkmark$ Vacuette tubes $(n=5)$ 
Figure 2:

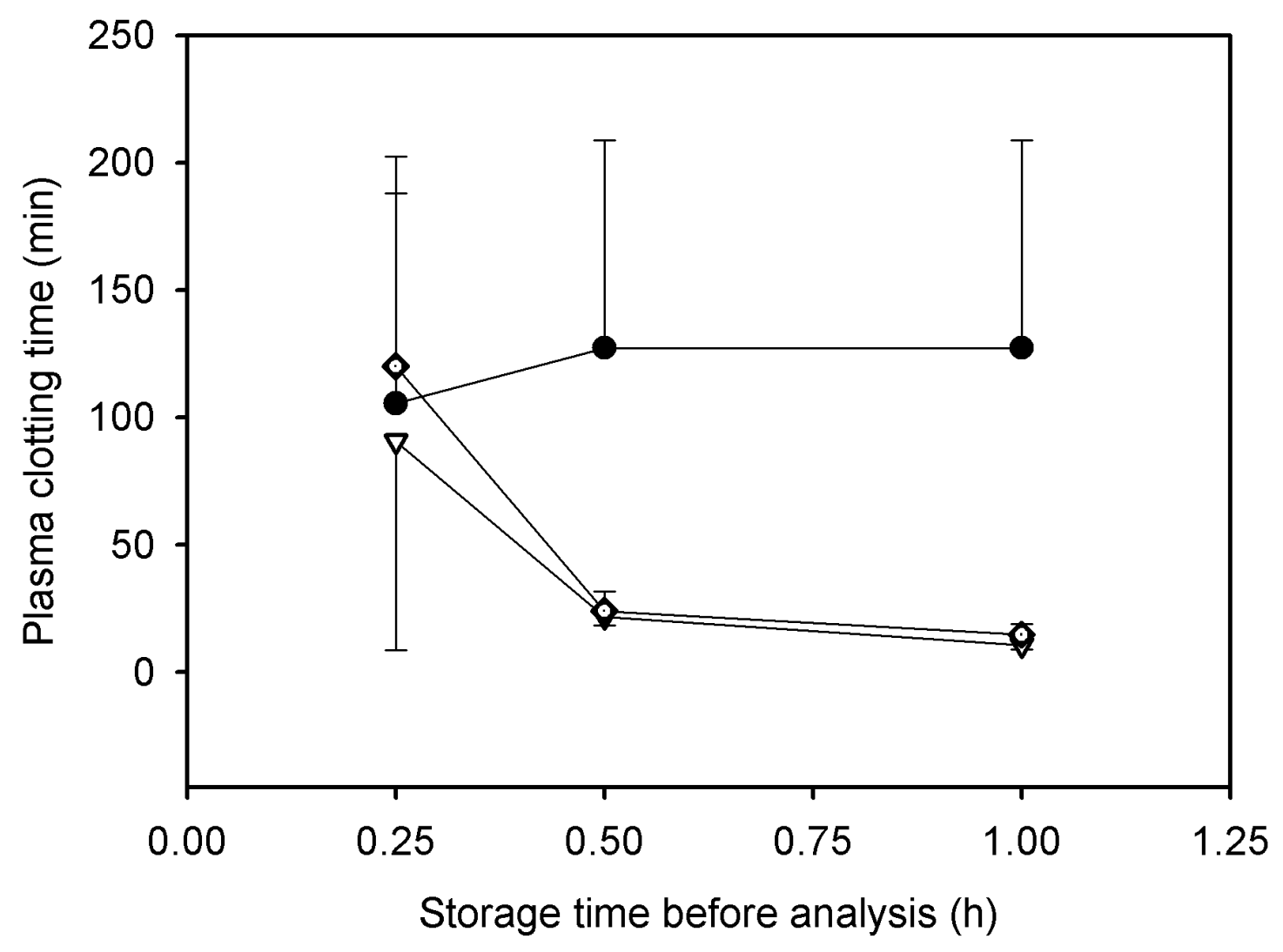

- Monovette tubes with Vacutainer citrate solution $(n=6)$
$-\frac{\nabla}{\sigma}-$ Vacutainer tubes $(n=5)$
- Vacuette tubes $(n=5)$ 
Figure 3:

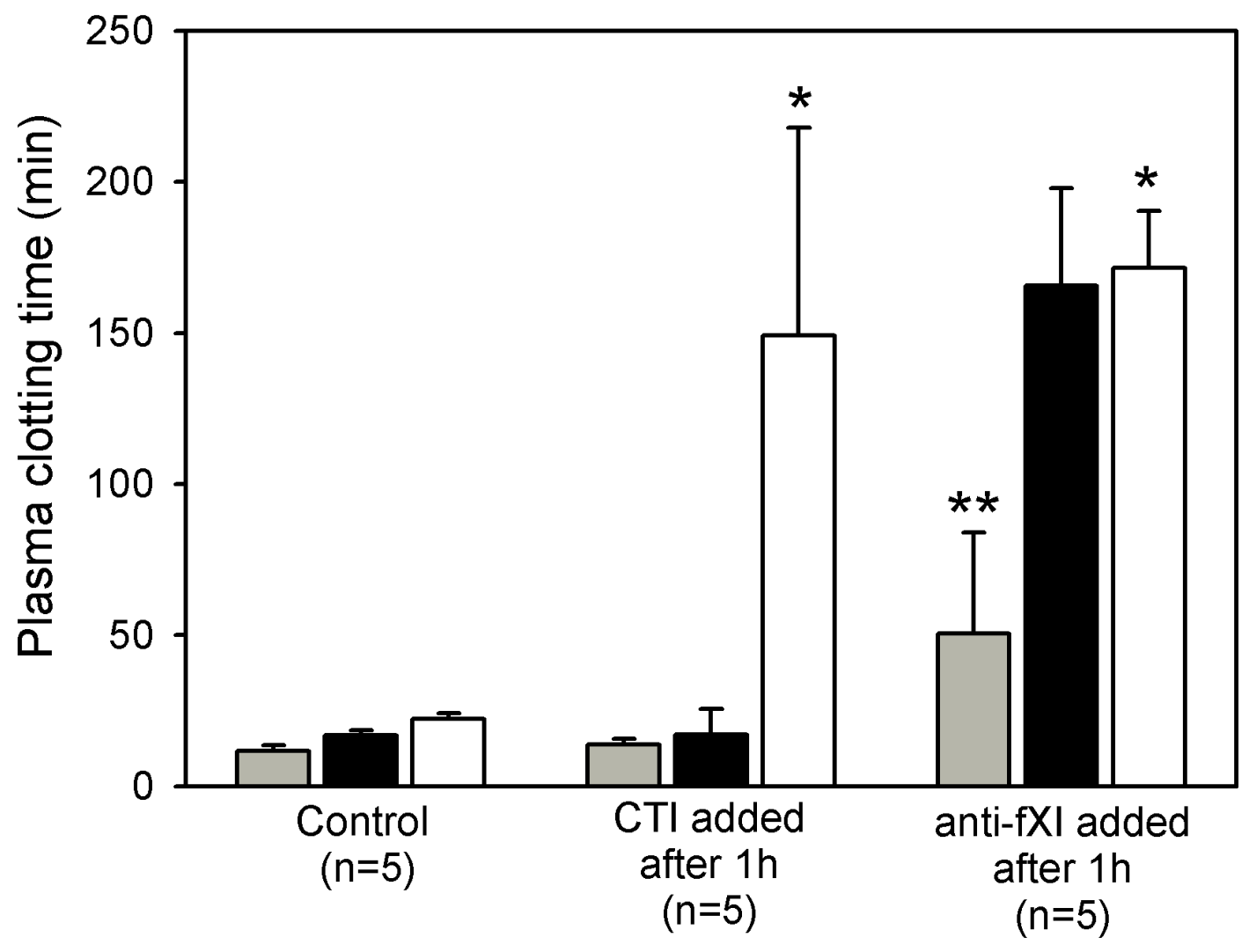

Vacutainer tubes

Vacuette tubes

$\square$ Monovette tubes with Vacutainer citrate solution 
Figure 4:

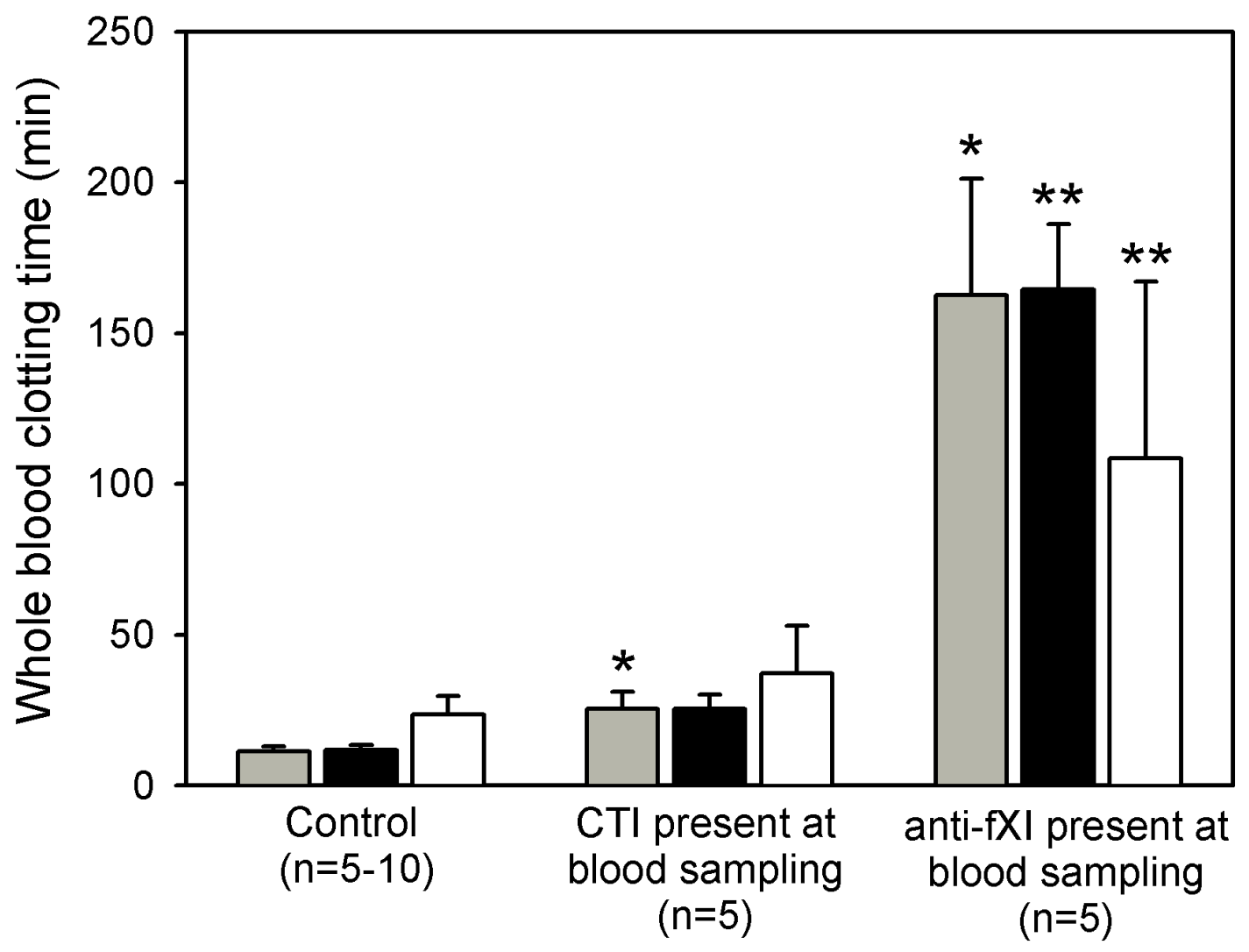

Vacutainer tubes

Vacuette tubes

Monovette tubes with Vacutainer citrate solution 
Figure 5:

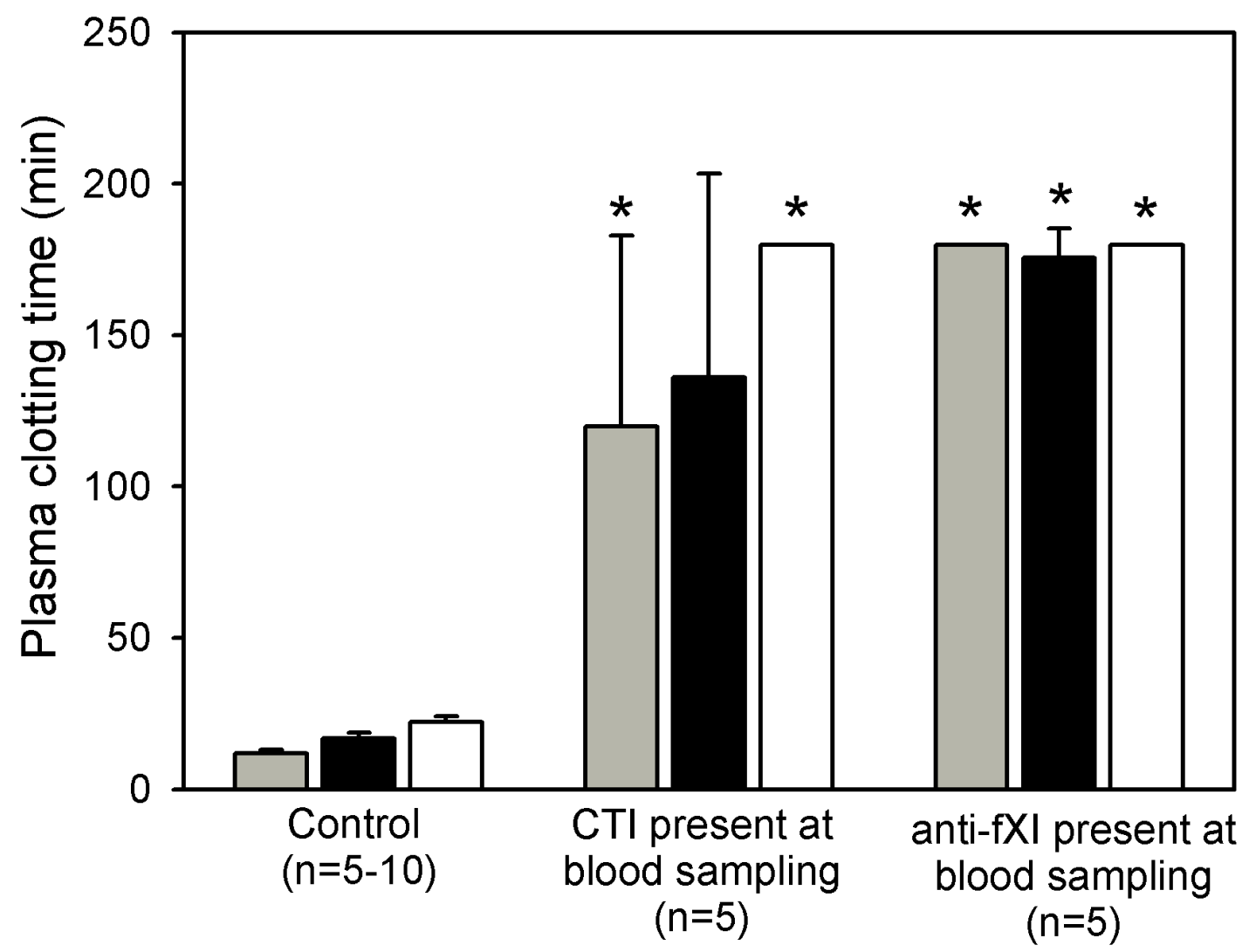

Vacutainer tubes

Vacuette tubes

$\square$ Monovette tubes with Vacutainer citrate solution 